\title{
FDA approves landmark RNAi drug
}

The FDA approved Alnylam's patisiran, the first drug to harness RNA interference (RNAi) to silence disease-associated gene expression.

The RNAi process was first discovered in 1998, and led to a Nobel prize for the discovering scientists in 2006. Whereas previously approved single-stranded oligonucleotides such as lonis Pharmaceuticals and Sanofi's mipomersen silence mRNA via antisense mechanisms, patisiran is a double-stranded small interfering RNA that ropes in the RNA-induced silencing complex to downregulate protein expression (see Nature's RNAi animation).

Patisiran reduces the expression levels of transthyretin (TTR), a transport protein that can misfold and aggregate to cause hereditary TTR-mediated amyloidosis, a rare, debilitating and often fatal genetic disease.

"Oligonucleotide therapeutics are going to represent the third major platform of drug development," Art Krieg, CEO of Checkmate Pharmaceuticals and former head of oligonucleotide therapeutics at Pfizer, told Nature Reviews Drug Discovery earlier this year. "At the beginning, I was skeptical about RNAi. But the progress in that field has been extraordinary."

At least six other RNAi therapeutics are already in phase III trials (TABLE 1). Whereas patisiran uses a lipid nanoparticle delivery mechanism to get the drug into cells in the liver, next-generation delivery approaches including $\mathrm{N}$-acetylgalactosamine (GalNAc)-conjugation stand to offer improved therapeutic indices and better side-effect profiles. RNAi-based drug developers hope that further work will enable efficient and effective delivery of these therapeutics into organs other than the liver and the eye.

Table 1 | Select late-stage RNA interference programmes

\begin{tabular}{|c|c|c|c|c|}
\hline Drug & Developer & Target & Indication & Status \\
\hline Patisiran & Alnylam & TTR & Hereditary ATTR amyloidosis & Approved \\
\hline QPI-1002 & Quark & p53 & Delayed graft function & Phase III \\
\hline QPI-1007 & Quark & Caspase 2 & $\begin{array}{l}\text { Non-arteritic anterior } \\
\text { ischaemic optic neuropathy }\end{array}$ & Phase III \\
\hline Tivanisiran & $\begin{array}{l}\text { Sylentis/ } \\
\text { PharmaMar }\end{array}$ & TRPV1 & Moderate to severe dry eye & Phase III \\
\hline Fitusiran & Sanofi/Alnylam & Antithrombin & $\begin{array}{l}\text { Haemophilia A and } \\
\text { haemophilia B }\end{array}$ & Phase III \\
\hline Givosiran & Alnylam & ALAS1 & Acute hepatic porphyrias & Phase III \\
\hline Inclisiran & $\begin{array}{l}\text { The Medicines } \\
\text { Company/Alnylam }\end{array}$ & PCSK9 & Hypercholesterolaemia & Phase III \\
\hline
\end{tabular}

Asher Mullard

\section{FDA approves second GPCR-targeted antibody}

Around one-third of approved small molecules act on $\mathrm{G}$ protein-coupled receptor (GPCR) targets, but these membrane proteins have proved harder to block with antibody-based drugs. With the FDA's August approval of Kyowa Hakko Kirin's mogamulizumab, the US regulators have now set the stage for the second GPCR-targeted antibody to make it to market.

Mogamulizumab acts on CC-chemokine receptor 4 (CCR4), a previously unexploited GPCR target. CCR4 is implicated in cancer, asthma and eczema, and plays a role in controlling the trafficking of immune cells. The surface receptor has stymied the efforts of small-molecule drug developers, but scientists at Kyowa Hakko Kirin found that their mogamulizumab binds to CCR4 on cancer cells, killing them via antibody-dependent cellular cytotoxicity.

The FDA approved the antibody for the treatment of two subtypes of cutaneous T cell lymphoma. In a pivotal trial of 372 patients with these subtypes, patients assigned to mogamulizumab had a median progression-free survival of 7.6 months, compared with 3.1 months for patients assigned to the histone deacetylase inhibitor vorinostat. (Japanese regulators approved the antibody in 2012 , based on open-label phase II data.)

Earlier this year, the FDA's approval of Amgen's CGRP inhibitor erenumab for the treatment of migraine marked the first US approval of a GPCR-targeted antibody.

At least ten other GPCR-targeted antibodies are in the clinic, thanks to lucky breaks, scientific advances in structural biology, technological progress in receptor engineering and new antibody-screening strategies (Nat. Rev. Drug Discov. 17, 457-459; 2018).

Asher Mullard

\section{Can you trust your cancer cell lines?}

By some estimates, as many as two-thirds of studies about new drug targets cannot be replicated owing to factors including statistical flaws, incomplete methodological descriptions, publication bias and outright fraud. Researchers have now added another irreproducibility culprit: genetic instabilities in cancer cell lines that cause seemingly standardized cells to behave differently from one another.

Todd Golub, CSO at the Broad Institute, and his colleagues analysed whole-exome sequences from 106 cell lines that were housed at both the Broad Institute and the Sanger Institute to explore their clonality and genetic stability. A median of $19 \%$ of the mutations they detected were only present in one set of paired cells.

In a deeper dive into whether these genetic inconsistencies translate into differences in drug responses, they administered 321 anticancer compounds to 27 strains of a common breast cancer cell line called MCF7. At least $75 \%$ of the compounds that blocked growth in some strains were completely inactive in others, they report in Nature.

"Established cancer cell lines, generally thought to be clonal, are in fact highly genetically heterogeneous," they conclude. "Cancer cell lines remain a powerful tool for cancer research, but their genomic evolution leads to a high degree of variation across cell line strains, which must be considered in experimental design and data interpretation."

Last year, Golub's team also showed that genetic inconsistencies can muddy the reliability of patient-derived xenograft mouse models of cancer, in which cancer cells are harvested from patients and injected into mice. Asher Mullard 


\section{FDA approves landmark RNAi drug}

doi:10.1038/nrd.2018.152

\section{Subject categories}

Biological sciences / Drug discovery

[URI /631/154]

Health sciences / Medical research / Drug development

[URI/692/308/153]

Biological sciences / Biotechnology

[URI /631/61]

Biological sciences / Chemical biology / Mechanism of action

[URI/631/92/609]

\section{Links}

Nature's RNAi animation

http://www.nature.com/nrg/multimedia/rnai/animation/index.html

told Nature Reviews Drug Discovery earlier this year

https://www.nature.com/articles/nrd.2018.20

Nat. Rev. Drug Discov. 17, 156-157; 2018

https://www.nature.com/articles/nrd.2018.20

FDA approves second GPCR-targeted antibody

doi:10.1038/nrd.2018.153

\section{Subject categories}

Biological sciences / Drug discovery

[URI/631/154]

Health sciences / Medical research / Drug development

[URI/692/308/153]

Biological sciences / Chemical biology / Mechanism of action

[URI/631/92/609]

Biological sciences / Drug discovery / Biologics / Antibody therapy

[URI /631/154/51/1568]

\section{Links}

Nat. Rev. Drug Discov. 17, 457-459; 2018

https://www.nature.com/articles/nrd.2018.103

\section{Can you trust your cancer cell lines?}

doi:10.1038/nrd.2018.154

\section{Subject categories}

Biological sciences / Drug discovery

[URI /631/154]

Health sciences / Medical research / Drug development

[URI /692/308/153]

Biological sciences / Drug discovery / Drug screening

[URI /631/154/1435]

Biological sciences / Chemical biology / Mechanism of action

[URI /631/92/609]

Biological sciences / Cancer / Cancer therapy / Drug development

[URI /631/67/1059/153]

\section{Links}

two-thirds of studies about new drug targets cannot be replicated https://www.nature.com/articles/nrd3545

report in Nature

https://www.nature.com/articles/s41586-018-0409-3 genetic inconsistencies can muddy the reliability of patient-derived xenograft mouse models

https://www.nature.com/articles/ng.3967 